\title{
Distribución y abundancia de Craugastor vulcani: una especie de rana en riesgo de Los Tuxtlas, Veracruz, México
}

\author{
Distribution and abundance of Craugastor vulcani: an endangered frog species from Los \\ Tuxtlas, Veracruz, Mexico
}

\author{
Eduardo Pineda ${ }^{1 *}$ y Clara A. Rodríguez-Mendoza ${ }^{1,2}$ \\ ${ }^{1}$ Red de Biología y Conservación de Vertebrados, Instituto de Ecología, A.C. Km. 2.5 carretera antigua a Coatepec 351, Congregación El Haya, \\ 91070 Xalapa, Veracruz, México. \\ ${ }^{2}$ Sierra Nuyuxia A. C. Calmecac 103-B Momoxpan, 72760 San Andrés Cholula, Puebla, México. \\ *Correspondencia: eduardo.pineda@inecol.edu.mx
}

\begin{abstract}
Resumen. En México, más de la mitad de las especies de anfibios están amenazadas y de la mayoría no se genera información que permita conocer el estatus en el que se encuentran sus poblaciones. En este trabajo se evaluó la distribución y abundancia de Craugastor vulcani, una rana endémica de la sierra de Los Tuxtlas Veracruz que se encuentra en riesgo de extinción, en función de la transformación de la selva. Para este trabajo, se consultaron bases de datos de registros históricos y se muestrearon 12 sitios que representan ambientes comunes de la región. Se registró un total de 524 individuos, de los cuales el $77 \%$ se encontraron en fragmentos de selva, el $20 \%$ en remanentes riparios y sólo el 3\% en potreros. La mayor abundancia (62\%) se registró durante la época seca del año. La distribución conocida de C. vulcani está sesgada hacia el norte de la sierra, existe poca información sobre su presencia en la porción sur. La permanencia de la especie en la zona parece depender de los pocos fragmentos de bosque existentes. El aumento en el número, área y conectividad de tales remanentes son algunas de las medidas necesarias para conservar ésta y otras especies amenazadas en la región.
\end{abstract}

Palabras clave: anfibios, especies en peligro, conservación, selva alta, transformación del hábitat.

\begin{abstract}
More than a half of all amphibian species occurring in Mexico are threatened, and for most of them there is no current information to assess the status of their populations. Craugastor vulcani is an endemic frog from the Los Tuxtlas mountains, Veracruz, and it is classified as an endangered species. We examine the distribution and abundance of $C$. vulcani as a function of rainforest transformation in Los Tuxtlas. We consulted historical records in databases and sampled 12 sites representing common habitats of the region. We recorded a total of 524 individuals of which $77 \%$ were found in rainforest fragments, $20 \%$ were collected in riparian remnants and just $3 \%$ in cattle pastures. Most individuals (62\%) were recorded during the dry season. The known distribution of $C$. vulcani is biased toward northern portion of the sierra, and there is little information about species occurrence in the southern part. Permanence of the species in the region seems to depend on the continued existence of the few forest fragment remnants. An increase in the number, area and connectivity of such forest fragments are some of the necessary actions to protect this and other threatened species in the studied region.
\end{abstract}

Key words: amphibians, endangered species, conservation, rainforest, habitat transformation.

\section{Introducción}

La disminución de poblaciones de anfibios es probablemente uno de los temas más críticos en el ámbito de la conservación biológica (Stuart et al., 2004). De las aproximadamente 6433 especies conocidas en todo el mundo (Frost, 2009) cerca de un tercio (32\%) se consideran amenazadas o extintas y de un $25 \%$ adicional no existe suficiente información que permita determinar su estatus

Recibido: 24 marzo 2009; aceptado: 05 julio 2009 de amenaza (IUCN, 2008). El mayor número de especies amenazadas se encuentra en países latinoamericanos, de los cuales México ocupa el segundo lugar con 211 especies de las 370 conocidas, sólo por detrás de Colombia con 214 especies amenazadas (IUCN, 2008). En la actualidad, la destrucción o modificación del hábitat se reconoce como la principal amenaza para los anfibios. Asimismo, la rápida emergencia de enfermedades infecciosas y la introducción de especies invasoras que actúan como depredadoras son también factores de relevancia que ponen en peligro la existencia de poblaciones de este 
grupo biológico (Lips et al., 2005).

Craugastor vulcani (Shannon and Werler, 1955) es una especie de rana microendémica de México cuya distribución se restringe a las selvas y bosques de niebla de la sierra de Los Tuxtlas, Veracruz (Frost, 2009). La especie, de talla grande, hábitos terrestres y con desarrollo directo está clasificada como especie bajo protección especial por la legislación mexicana (SEMARNAT, 2002, listada como Eleutherodactylus berkenbuschi, por su anterior sinonimia: Campbell y Savage, 2000) y en peligro de extinción en la Lista Roja de la UICN 2008 (Fig. 1). Debido a que su área de distribución es menor de $5000 \mathrm{~km}^{2}$, todos los individuos se encuentran en menos de 5 localidades y existe una continua disminución del área y calidad de su hábitat (Santos-Barrera, 2004). Asimismo, no se tiene conocimiento sobre el estatus de sus poblaciones pero se estima que están en descenso y, aunque hace más de una década se informó que $C$. vulcani era abundante en la selva que rodea la Estación de Biología Los Tuxtlas (Vogt et al., 1994), se desconoce cuál es su situación actual y si la especie puede habituarse a las transformaciones del hábitat (Santos-Barrera, 2004).
En la sierra de Los Tuxtlas, Veracruz, la deforestación es la causa de la pérdida del $78 \%$ del bosque original, la mayor parte del paisaje se convirtió en pastizales para ganado y sólo permanece poco más de 54000 ha de cubierta forestal (evaluaciones hasta 1993 para toda la sierra; véase Guevara et al., 2004a). La mayor proporción del bosque que aún persiste se encuentra por encima de los 800 m hacia las cimas de los volcanes San Martín Tuxtla, Santa Marta y San Martín Pajapan (Estrada y CoatesEstrada, 1996; Guevara et al., 2004a). Este hecho pone de manifiesto que la selva alta es el tipo de vegetación más severamente transformado, la cual se ubica originalmente en bajas altitudes, lo que ha dado lugar a un paisaje que se conforma por un conjunto de fragmentos de selva inmersos en un mosaico de pastizales para ganado y otros ambientes transformados (Dirzo y García, 1992; Estrada y Coates-Estrada, 1996, Guevara et al., 2004). El mosaico de hábitats transformados puede funcionar como filtro selectivo del movimiento de las especies a través del paisaje, algunos organismos del bosque pueden usar hábitats de dicho mosaico para desplazarse o reproducirse pero otros son incapaces de utilizarlo, en este último caso,

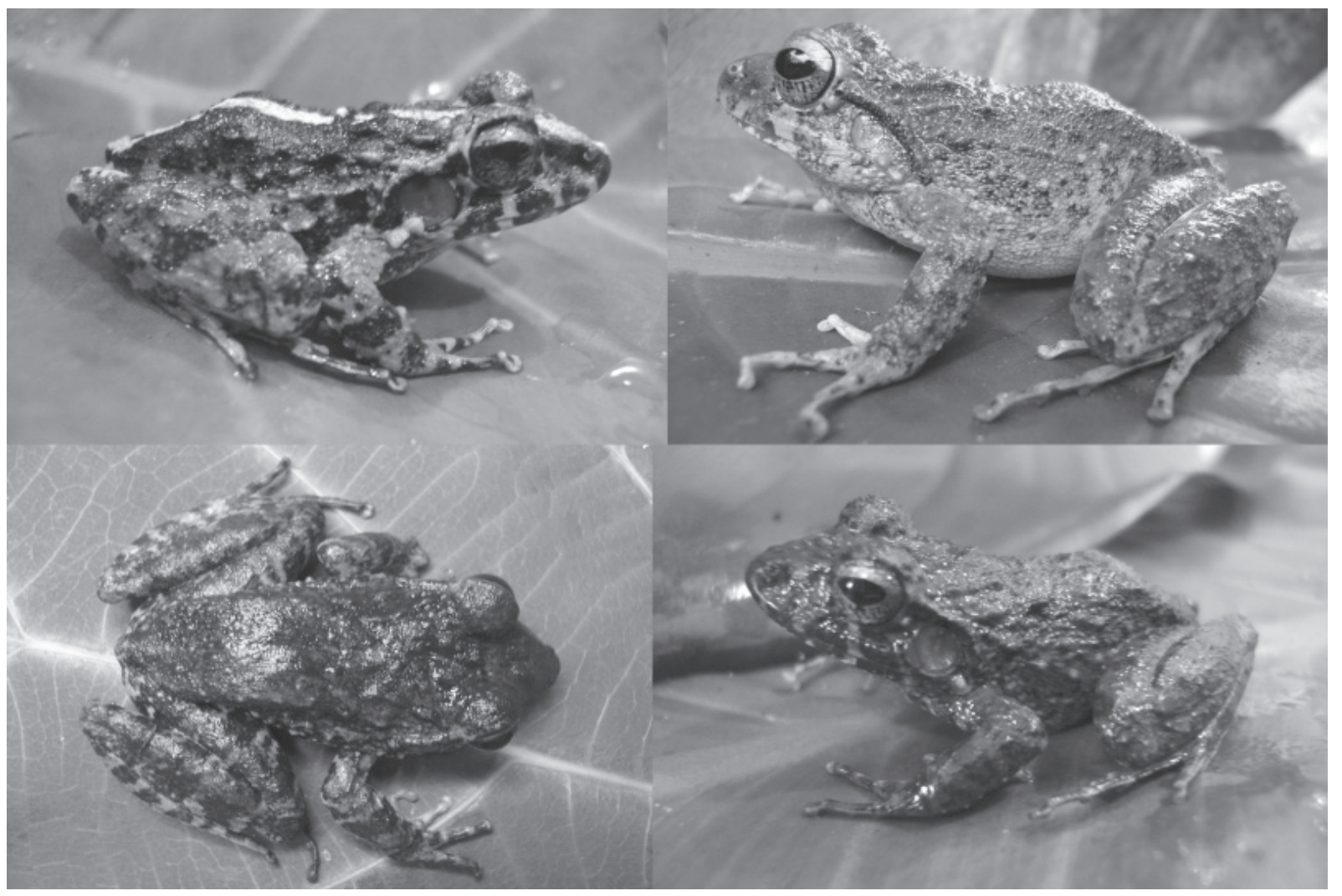

Figura 1. Craugastor vulcani. Variación morfológica de los individuos recolectados en el presente estudio (fotos: C. A. RodríguezMendoza). 
el mosaico actúa como barrera y se percibe como un ambiente inhóspito (Gascon et al., 1999; Estrada, 2008).

Diversos estudios en bosques tropicales documentan los efectos negativos de la pérdida y fragmentación del hábitat sobre las comunidades o poblaciones de anfibios; sin embargo, para algunas especies el efecto parece ser neutro o incluso positivo, ya que su abundancia se mantiene constante, o bien, aumenta, esto sugiere que algunas especies son capaces de habituarse a ambientes con fuerte transformación (Pineda y Halffter, 2004; Urbina-Cardona et al., 2006; Gardner et al., 2007; Suazo-Ortuño et al., 2008). Si la transformación y fragmentación del hábitat tiene un efecto diferencial sobre las especies de anfibios, resulta entonces relevante conocer cómo influye este fenómeno sobre C. vulcani, considerada en peligro de extinción. En particular, el propósito de este estudio fue evaluar cómo es la distribución y abundancia de $C$. vulcani en función de la transformación de la selva en un paisaje de Los Tuxtlas. Para ello, se examinó la distribución conocida de la especie en la región y se evaluó tanto su presencia como su abundancia en relación con el tipo de hábitat: selva o potrero, en un paisaje de la porción norte de la sierra. Mediante este trabajo se pretende ofrecer información actual que contribuya a una mejor comprensión del estado que guarda $C$. vulcani frente a un fenómeno creciente en la región como es la deforestación.

\section{Materiales y métodos}

Área de estudio. La sierra de Los Tuxtlas se ubica al sur del estado de Veracruz (Fig. 2). Su origen es volcánico, posee un accidentado relieve y un gradiente altitudinal que va desde el nivel del mar hasta los $1680 \mathrm{~m}$ snm. La sierra se caracteriza por su aislamiento de cualquier otro sistema montañoso y su extensión es cercana a los 3300 $\mathrm{km}^{2}$; en ella se encuentra la Reserva de la Biosfera Los Tuxtlas (Guevara et al., 2004b). El clima predominante es cálido-húmedo con temperatura media anual entre los $24^{\circ} \mathrm{C}$ y $26{ }^{\circ} \mathrm{C}$ y la precipitación anual oscila entre 3000 y $4500 \mathrm{~mm}$; aunque llueve durante todo el año, existe una marcada estacionalidad donde la época húmeda va de junio a febrero y la seca de marzo a mayo (Soto, 2004). Dada su ubicación geográfica, historia geológica y alta heterogeneidad ambiental, la sierra es diversa en tipos de vegetación. En ella es posible distinguir al menos 9 tipos, entre los que destacan la selva alta perennifolia, la selva mediana perennifolia, el bosque mesófilo de montaña y los bosques de pino y encino, además de los sistemas agrícolas derivados (Castillo-Campos y Laborde, 2004).

Paisaje y sitios de estudio. El trabajo de campo se ubicó en un paisaje al norte de la sierra (Fig. 1). El área, cuyo intervalo altitudinal oscila entre el nivel del mar y los $450 \mathrm{~m} \mathrm{snm}$, originalmente estuvo cubierta por selva alta perennifolia y en la actualidad está formada por múltiples fragmentos de selva que en conjunto ocupan aproximadamente el 24\% del área (Arroyo-Rodríguez et al., 2009); el resto de la superficie son potreros y otros sistemas agrícolas. El paisaje se ubica dentro de la reserva de la biosfera e incluye la Estación de Biología Los Tuxtlas del Instituto de Biología, UNAM. Entre los fragmentos de selva es posible distinguir aquellos de forma irregular y tamaño variable, de aquellos con forma alargada, área pequeña y que comúnmente flanquean un río o arroyo, conocidos
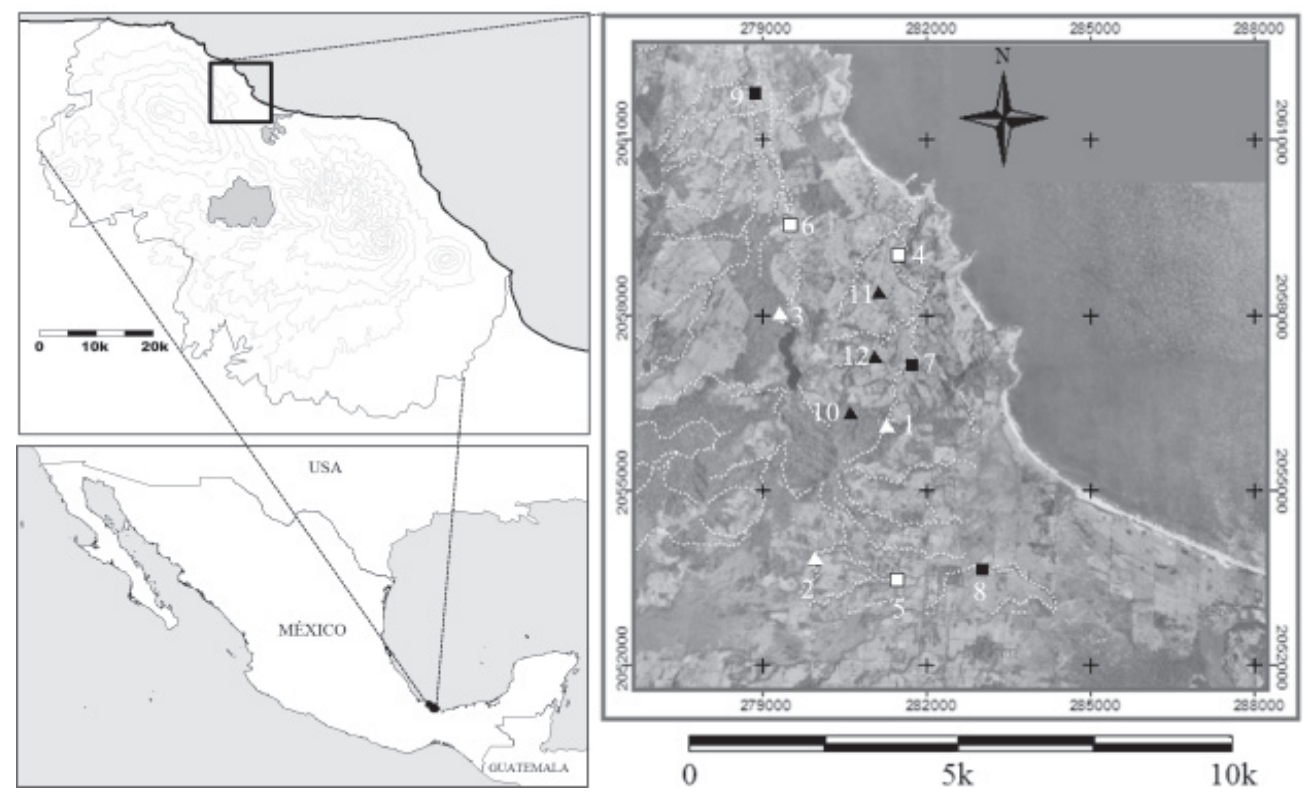

Figura 2. Ubicación de la sierra de Los Tuxtlas, del paisaje y de los sitios de estudio. Los triángulos blancos en la fotografía aérea denotan la ubicación de los sitios de estudio en fragmentos de selva, los cuadros blancos indican sitios en riparios, los cuadros negros son sitios en potreros y los triángulos negros son sitios sin río. El número indica la clave de cada sitio (véase Cuadro 1). 
también como fragmentos riparios (para los propósitos de este estudio se denominan fragmentos y riparios, respectivamente). Los potreros, el sistema predominante en la zona, en general están delimitados por una fila de árboles, llamada "cerca viva", y mantienen en el interior árboles aislados que proveen sombra al ganado.

Para seleccionar los sitios de estudio se siguieron los siguientes criterios: 1) que representasen un ambiente común del paisaje; 2) que contuvieran un río o arroyo, y 3) que la distancia mínima entre ellos fuese superior a 500 m. De esta manera, se seleccionaron 9 sitios ubicados en 3 ambientes: 3 sitios en fragmentos de selva, 3 en fragmentos riparios y 3 en potreros. Adicionalmente, se seleccionaron 3 sitios que ambientalmente fuesen semejantes a los sitios previamente seleccionados, pero que no tuviesen río. Esto con el propósito de evaluar la importancia de los cuerpos de agua lóticos para la presencia de $C$. vulcani que, aunque su forma de reproducción es el desarrollo directo y la larva no pasa por una etapa acuática, su presencia se ha vinculado con la cercanía de ríos o arroyos (Ramírez-Bautista, 1977). Los sitios sin río fueron un fragmento de selva, una cerca viva y un potrero, los cuales, en conjunto con los 9 sitios seleccionados previamente, resultaron en 12 sitios de estudio (Fig. 2). Cada sitio se caracterizó ambientalmente y se determinó su tamaño, altitud, cobertura del dosel, porcentaje de hojarasca, temperatura y humedad relativa. Las variables ambientales se obtuvieron en el lugar donde se trazaron los transectos de muestreo de ranas (véase más adelante).

Acopio de datos. El acopio de datos biológicos tuvo 2 etapas, una fue la consulta de bases de datos especializadas para examinar la distribución conocida de la especie en la región, y la otra consistió en el muestreo en campo para verificar la presencia de $C$. vulcani y evaluar su abundancia en función del tipo de ambiente.

Consulta de bases de datos. Para examinar la distribución conocida de $C$. vulcani en la región se consultaron 3 fuentes de información de las cuales se pudiesen obtener registros georreferenciados. Las fuentes de consulta fueron el Sistema Nacional de Información Biológica de la CONABIO, la base de datos de Global Biodiversidty Information Facility (www.gbif.org) y Herpnet (www. herpnet.org). Las palabras clave para la búsqueda fueron Craugastor vulcani, Craugastor (o Eleutherodactylus) berkenbuschii y Craugastor (o Eleutherodactylus) rugulosus. Se buscó información sobre berkenbuschii y rugulosus, ya que Campbell y Savage (2000), después de hacer una serie de reconsideraciones taxonómicas del grupo Eleutherodactylus de Mesoamérica, removieron a E. vulcani de E. berkenbuschii (originalmente considerada como parte de E. rugulosus por Lynch, 1965), en particular para los ejemplares de la región de Los Tuxtlas. De los registros que se obtuvieron se seleccionaron aquellos de la región de Los Tuxtlas, de acuerdo con la propuesta de Campbell y Savage (2000). Para proyectar los registros georreferenciados de C. vulcani en el espacio geográfico se usó el programa DIVA-GIS versión 5.2 (Hijmans et al., 2001).

Muestreo de ranas. Para registrar la presencia y abundancia de C. vulcani en cada sitio de estudio se trazó de manera paralela al río un transecto de $900 \mathrm{~m}^{2}(180 \times 5$ $\mathrm{m}$ ); sólo en los sitios sin río la dirección del transecto fue al azar. Cada transecto se muestreó mediante búsquedas directas y encuentros visuales (Crump y Scott, 1994) por 3 personas durante una hora, en 8 ocasiones o visitas. Éstas se llevaron a cabo en los meses de junio, agosto y noviembre de 2007 y abril de 2008 (2 visitas por mes) en horario nocturno. Así, el esfuerzo de muestreo por sitio fue de 24 horas-persona ( 8 visitas $\times 3$ personas $\times 1$ hora) y el esfuerzo en todo el paisaje fue de 288 horas-persona (12 sitios $\times 24$ horas-persona). Al aplicar un esfuerzo de muestreo medido en tiempo y estandarizado a una misma área de búsqueda por sitio, en este caso $900 \mathrm{~m}^{2}$, se reduce el posible efecto que los distintos tamaños de fragmento o de potrero puedan tener sobre la probabilidad de detección y abundancia registrada. Los muestreos de abril y junio fueron durante la época seca (en el año 2007 el inicio de la época de lluvias se retrasó hasta julio), mientras que los muestreos de agosto y noviembre se realizaron en la época lluviosa. De los individuos recolectados, sólo 3 se conservaron en alcohol al $70 \%$ como ejemplares testigo, los restantes se liberaron en el mismo sitio de recolecta y en algunos casos se tomaron fotografías digitales. Para tener una idea de la edad relativa de los individuos en estudio, a cada organismo se le midió la longitud hocico-cloaca (LHC) y la masa corporal. En el caso de los individuos que se reconocieron visualmente sólo se anotó el número de ejemplares observados.

Análisis de datos. Con el propósito de detectar alguna asociación de la abundancia de $C$. vulcani con atributos locales (de los sitios) se eligieron 6 variables para caracterizar a cada sitio: 1) altitud; 2) área del fragmento, ripario o potrero en el que se encuentra cada transecto; 3 ) cobertura del dosel; 4) cantidad de hojarasca; 5) temperatura atmosférica; y 6) humedad relativa. La altitud se obtuvo con un altímetro Thommen. El área se estimó mediante el análisis de fotografías aéreas digitales (INEGI, 1999) con el programa ArcView 3.2. La cobertura del dosel se midió a través de fotografías digitales tomadas en 10 puntos (separados cada $20 \mathrm{~m}$ ) en cada sitio. Posteriormente, las imágenes se analizaron con el programa ImageJ 1.37 (Rasband, 1997). Para medir la cantidad de hojarasca en el suelo se estimó el porcentaje de hojarasca en 4 cuadros de $25 \times 25 \mathrm{~cm}$ en 10 puntos de muestreo (los mismos puntos 
usados para medir cobertura del dosel). La temperatura atmosférica y la humedad relativa se registraron al inicio y final de cada transecto, durante todos los muestreos, con una estación meteorológica portátil Kestrel 3000. El Cuadro 1 muestra las características de los 12 sitios estudiados.

Para detectar aquellas variables locales que pudieran estar relacionadas con la abundancia de C. vulcani se efectuó un análisis de regresión múltiple paso a paso mediante el uso del programa STATISTICA 7.0 (StatSoft, 2004) previa transformación de datos mediante raíz cuadrada (Zar, 1999). Se empleó una prueba de $\chi^{2}$ para evaluar si la abundancia registrada de $C$. vulcani era diferente entre ambientes, así como entre época seca y época de lluvias (Zar, 1999).

\section{Resultados}

Un total de 524 individuos de $C$. vulcani fueron registrados en el paisaje de estudio. No se detectaron individuos de $C$. vulcani en 4 de los 12 sitios estudiados (los 3 lugares sin río y el sitio 8: potrero). En el resto de los sitios, la abundancia osciló entre 190 individuos (sitio 1: fragmento) y un individuo (sitio 9: potrero, Cuadro 2). La abundancia registrada entre ambientes fue significativamente diferente $\left(\chi^{2}=477, \mathrm{p}<0.01\right)$ donde el conjunto de fragmentos de selva tuvo el mayor número de individuos con 404, en el conjunto de riparios se detectaron 107 individuos y en 2 potreros se registró un acumulado de 13 ejemplares. En los 3 sitios ubicados en fragmentos de selva se registraron ejemplares durante todos los muestreos, en los riparios no hubo recolectas en 1 o 2 ocasiones, mientras que en los 2 potreros no se registraron individuos en 1 y en 7 ocasiones, en ambos casos con abundancias muy bajas (Cuadro 2). Por otro lado, no se encontró asociación alguna entre la abundancia observada de cada sitio y las variables locales medidas $\left(\mathrm{F}_{6,5}=1.149\right.$, $\mathrm{R}^{2}=0.579, \mathrm{p}=0.449$ ).

El número de individuos que se registraron entre épocas del año difirió significativamente $\left(\chi^{2}=30.3, \mathrm{p}<\right.$ $0.01)$ ya que de los 524 ejemplares registrados en total, el $62 \%$ (325 individuos) se detectó en época seca, y el $38 \%$ (199 individuos) en época de lluvias (Cuadro 2). A excepción de los potreros, en todos los sitios se observó mayor abundancia durante la época seca.

La variación en el tamaño y masa de los individuos recolectados (456) fue notable. El tamaño (LHC) osciló entre $12 \mathrm{~mm}$ y $73 \mathrm{~mm}$ con un promedio de $35.5 \mathrm{~mm}$. Dos terceras partes de los individuos recolectados (306) midieron menos de $40 \mathrm{~mm}$, mientras que sólo el 10\% (47) midió más de $60 \mathrm{~mm}$ (Fig. 3a). El peso de los ejemplares medidos varió entre $0.1 \mathrm{~g}$ y $47.4 \mathrm{~g}$ con un promedio calculado de 7.5 g. El $85 \%$ de los individuos medidos tuvieron un peso inferior a $12 \mathrm{~g}$, mientras que únicamente el $4 \%$ pesó más de $35 \mathrm{~g}$ (Fig. 3b).

En las bases de datos de CONABIO, GBIF y Herpnet se encontraron 119 registros de $C$. vulcani distribuidos en

Cuadro 1. Características de los 12 sitios estudiados en un paisaje de la Sierra de Los Tuxtlas, Veracruz, México

\begin{tabular}{|c|c|c|c|c|c|c|c|}
\hline Ambiente & $\begin{array}{l}\text { Sitio } \\
\text { (clave) }\end{array}$ & $\begin{array}{l}\text { Altitud } \\
\text { (msnm) }\end{array}$ & $\begin{array}{l}\text { Área* } \\
\text { (ha) }\end{array}$ & $\begin{array}{l}\text { Cobertura del } \\
\text { dosel }(\% \pm D E)\end{array}$ & $\begin{array}{c}\text { Hojarasca } \\
(\% \pm D E)\end{array}$ & $\begin{array}{l}\text { Temperatura } \\
\left({ }^{\circ} \mathrm{C} \pm D E\right)\end{array}$ & $\begin{array}{l}\text { Humedad relativa } \\
(\% \pm D E)\end{array}$ \\
\hline \multirow{3}{*}{ Fragmento } & 1 & 145 & 27.6 & $84.3 \pm 1.7$ & $60.1 \pm 6.2$ & $25.2 \pm 1.6$ & $81.0 \pm 11.1$ \\
\hline & 2 & 90 & 3.7 & $83.4 \pm 2.0$ & $69.4 \pm 17.3$ & $25.5 \pm 2.9$ & $79.3 \pm 10.6$ \\
\hline & 3 & 150 & 197.4 & $81.9 \pm 5.4$ & $56.9 \pm 10.9$ & $26.5 \pm 1.8$ & $81.8 \pm 12.9$ \\
\hline \multirow{3}{*}{ Ripario } & 4 & 25 & 3.0 & $81.1 \pm 2.3$ & $22.8 \pm 16.8$ & $26.0 \pm 2.5$ & $82.8 \pm 9.4$ \\
\hline & 5 & 30 & 0.4 & $85.0 \pm 0.7$ & $73.5 \pm 11.1$ & $24.8 \pm 3.0$ & $83.1 \pm 11.8$ \\
\hline & 6 & 35 & 0.7 & $79.1 \pm 5.8$ & $59.5 \pm 9.5$ & $26.6 \pm 3.3$ & $79.6 \pm 12.7$ \\
\hline \multirow{3}{*}{ Potrero } & 7 & 45 & 9.4 & $39.3 \pm 16.9$ & $15.1 \pm 5.6$ & $25.5 \pm 2.3$ & $80.4 \pm 11.8$ \\
\hline & 8 & 15 & 24.7 & $9.4 \pm 3.9$ & $3.0 \pm 4.1$ & $23.3 \pm 4.0$ & $81.7 \pm 9.3$ \\
\hline & 9 & 10 & 14.3 & $10.9 \pm 9.7$ & $4.4 \pm 6.9$ & $25.3 \pm 2.1$ & $83.4 \pm 8.6$ \\
\hline \multirow{3}{*}{ Sin río } & 10 & 215 & 266.0 & $86.7 \pm 3.7$ & $73.8 \pm 5.3$ & $24.8 \pm 1.8$ & $86.3 \pm 13.2$ \\
\hline & 11 & 50 & 1.6 & $77.8 \pm 4.8$ & $54.7 \pm 3.4$ & $25.1 \pm 3.2$ & $80.6 \pm 8.1$ \\
\hline & 12 & 60 & 3.7 & $6.4 \pm 4.5$ & $1.8 \pm 1.0$ & $25.8 \pm 1.8$ & $80.2 \pm 12.4$ \\
\hline
\end{tabular}

*Área= Extensión del fragmento, ripario o potrero donde se ubicó cada transecto; Los sitios 10, 11 y 12 corresponden a los ambientes sin río: fragmento, cerca viva y potrero, respectivamente DE= Desviación estándar. 
Cuadro 2. Número de individuos de Craugastor vulcani registrados en los sitios estudiados, así como por época del año en un paisaje de la Sierra de Los Tuxtlas, Veracruz, México

\begin{tabular}{|c|c|c|c|c|c|c|c|c|c|c|c|c|}
\hline \multirow[b]{2}{*}{ Sitio $^{*}$} & \multicolumn{8}{|c|}{ Muestreos** } & \multicolumn{2}{|c|}{ Época } & \multirow{2}{*}{$\begin{array}{c}\text { Total por } \\
\text { sitio }\end{array}$} & \multirow{2}{*}{$\begin{array}{l}\text { Total por } \\
\text { ambiente }\end{array}$} \\
\hline & 1 & 2 & 3 & 4 & 5 & 6 & 7 & 8 & Seca & Lluvias & & \\
\hline 1 & 3 & 8 & 26 & 32 & 10 & 17 & 33 & 61 & 105 & 85 & 190 & \\
\hline 2 & 7 & 6 & 3 & 7 & 4 & 1 & 6 & 34 & 53 & 15 & 68 & \\
\hline 3 & 25 & 15 & 15 & 19 & 9 & 5 & 23 & 35 & 98 & 48 & 146 & $404^{\mathrm{F}}$ \\
\hline 4 & 1 & & 3 & 3 & 4 & & 9 & 6 & 16 & 10 & 26 & \\
\hline 5 & 4 & & 4 & & 1 & 1 & 4 & 2 & 10 & 6 & 16 & \\
\hline 6 & & 2 & 9 & 13 & 2 & 3 & 13 & 23 & 38 & 27 & 65 & $107^{\mathrm{R}}$ \\
\hline 7 & 2 & & 2 & 3 & 1 & 1 & & 3 & 5 & 7 & 12 & \\
\hline 9 & & & 1 & & & & & & & 1 & 1 & $13^{\mathrm{P}}$ \\
\hline Total & 42 & 31 & 63 & 77 & 31 & 28 & 88 & 164 & 325 & 199 & 524 & 524 \\
\hline
\end{tabular}

*Sólo en 8 de los 12 sitios estudiados se registró la especie.

** Los muestreos 1, 2, 7 y 8 corresponden a la época seca, los muestreos del 3 al 6 corresponden a la época de lluvias. Los superíndices F, R, y P indican los ambientes fragmento, ripario y potrero, respectivamente.

45 localidades. Un registro significa 1 o más individuos de la misma especie en una sola localidad y fecha de recolecta. $\mathrm{Al}$ incorporar nuestros datos de campo (8 localidades y 50 registros) a la base de datos general, resultó un total de 169 registros y 53 localidades. Al proyectar los registros en el espacio geográfico se observa que su distribución tiende a estar agregada hacia el norte de la sierra (Fig. 4). En el área del volcán Santa Marta (zona centro-sur) hay muy pocos registros y en la zona del volcán San Martín Pajapan (sureste) existen 3 localidades de recolecta. Las fechas en que se ha recolectado C. vulcani van desde 1953 hasta 2008; su distribución altitudinal oscila entre el nivel del mar y los 1400 m snm (hacia la cima del volcán San Martín Tuxtla). Los ejemplares recolectados desde 1953 a la fecha se encuentran depositados en al menos 10 colecciones científicas: 3 nacionales y 7 extranjeras.

\section{Discusión}

Nuestros resultados indican que $C$. vulcani es notablemente sensible a la modificación del hábitat y no es capaz de habituarse a ambientes transformados como los potreros. En el paisaje estudiado la especie es abundante, aunque su presencia se restringe fundamentalmente a los fragmentos de selva que contienen cuerpos de agua. Su distribución en la sierra, desde el nivel del mar hasta los $1400 \mathrm{~m}$ snm, está mejor documentada hacia la porción norte, mientras que en la parte sur el número de registros es limitado. La permanencia de la especie en la región parece depender de los pocos fragmentos de bosque que aún existen.

Las abundancias altas y moderadas de $C$. vulcani que se detectaron en los fragmentos de selva y los remanentes riparios, respectivamente, sugieren que la especie es capaz de mantenerse adecuadamente dentro de los remanentes de vegetación, particularmente en aquellos que contienen cuerpos de agua. Sin embargo, fuera de estos fragmentos, se observan muy pocos individuos o incluso no es posible detectarlos. En este sentido, la matriz de hábitats transformados, dominada por pastizales para ganado, parece funcionar como una barrera para el movimiento de los individuos de $C$. vulcani en el paisaje y operar como un ambiente hostil para su sobrevivencia y/o reproducción.

La alta abundancia de la especie (en ocasiones registrada como Eleutherodactylus berkenbuschii o E. rugulosus) en ambientes de selva ha sido advertida a lo largo de varios años en la zona de estudio. RamírezBautista (1977) menciona que la especie es relativamente abundante en la zona, principalmente en selva semiabierta, observándose con mayor facilidad a orillas de los arroyos y charcas.Vogt et al. (1997) informan, 2 décadas después, que la especie es abundante en el bosque de la Estación de Biología, sin mencionar cifras. Hernández (2005) al comparar la comunidad de anfibios y reptiles entre zonas altas y bajas de la reserva de la Estación de Biología registra 117 individuos de $C$. vulcani, considerándola la segunda especie de anfibio más abundante, ubicados todos ellos hacia la parte baja en los alrededores del río. Por su parte, Salvatore (2006), al analizar la estructura de la comunidad 

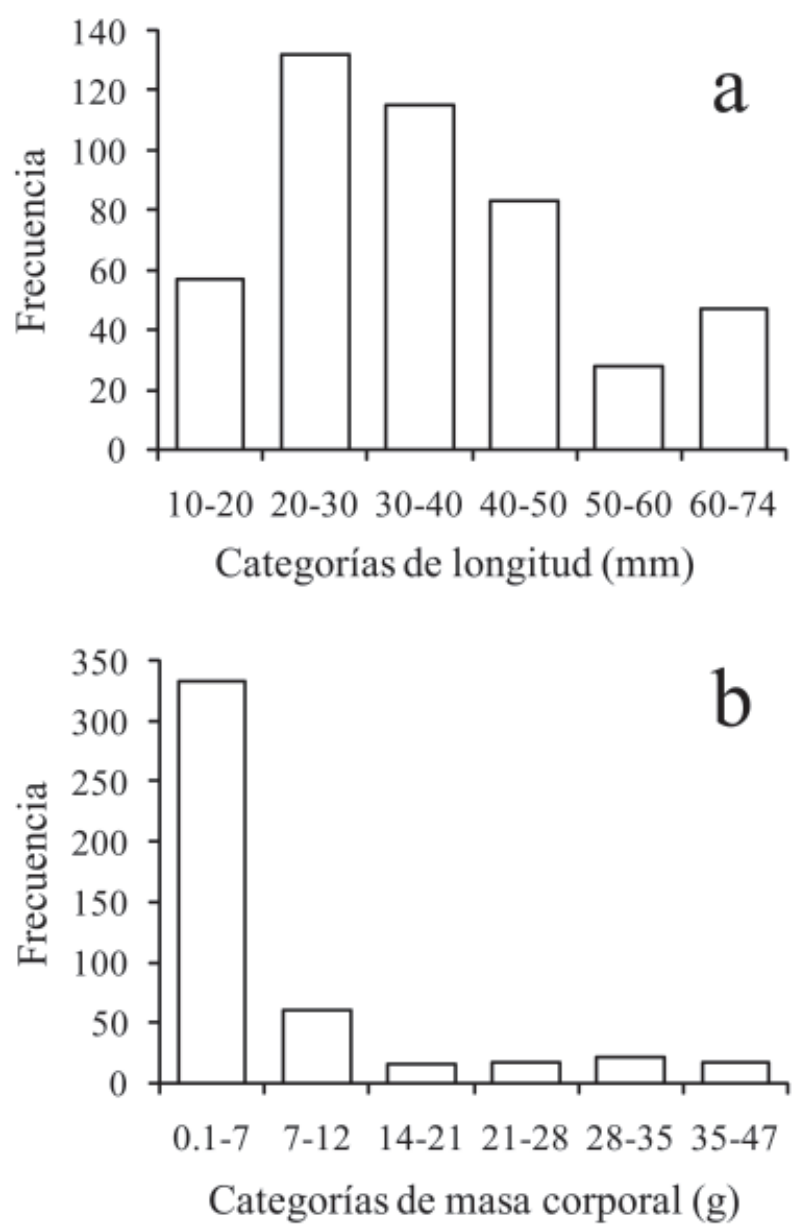

Figura 3. Distribución de frecuencias por categorías de longitud y categorías de masa corporal de 465 individuos de Craugastor vulcani recolectados y liberados en un paisaje al norte de la sierra de Los Tuxtlas, Veracruz.

de herpetozoos en fragmentos de selva, bosques secundarios y potreros en los alrededores de la Estación, detecta 182 individuos de C. vulcani distribuidos en 4 fragmentos de selva y 1 fragmento de selva secundaria (sólo 1 individuo en este último). En la misma zona, Villar (2007) examina la composición y diversidad de anfibios y reptiles en áreas con características de corredores biológicos y registra 27 individuos en 6 de los 8 sitios muestreados (oscilando entre 1 y 12 individuos por sitio).

En este estudio se remarca la fuerte asociación entre la presencia de $C$. vulcani y la existencia de cuerpos de agua en los fragmentos de vegetación. Aunque el modo reproductivo de la especie es por desarrollo directo, es decir, la larva no pasa por etapa acuática, parece que el microclima y la oferta de recursos asociada a los ríos y arroyos desempeñan

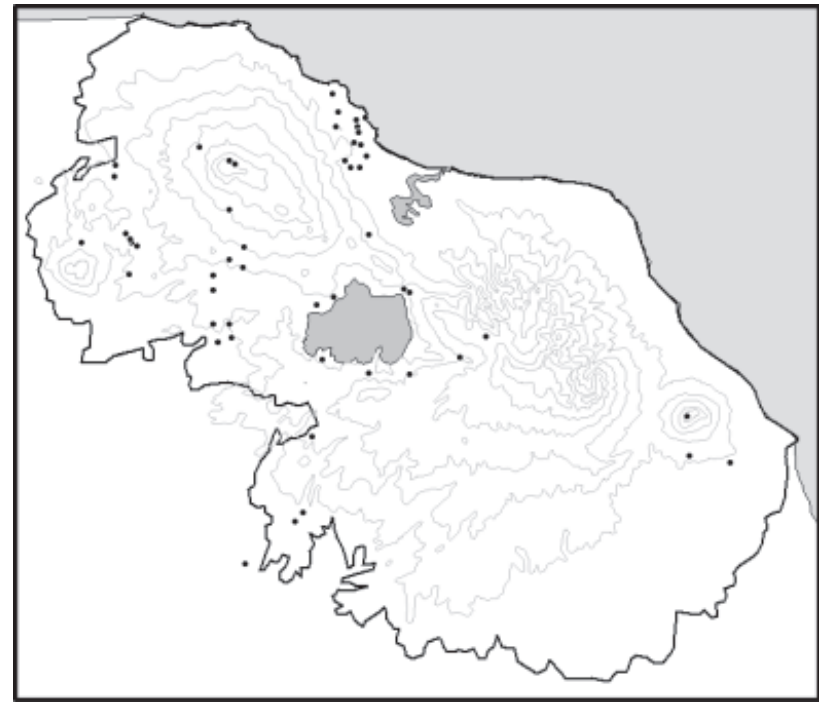

Figura 4. Distribución de las localidades de recolecta de Craugastor vulcani en la sierra de Los Tuxtlas, Veracruz. Los puntos indican las localidades donde se ha recolectado la especie desde 1953, la línea gruesa señala los límites aproximados de la sierra y las líneas delgadas denotan curvas de nivel cada $200 \mathrm{~m}$. El Lago de Catemaco y la Laguna de Sontecomapan se muestran en la parte central de la imagen.

un papel importante en la sobrevivencia y/o reproducción de C. vulcani. La sola existencia de vegetación de selva o bosque no parece suficiente para mantener un alto número de individuos. Urbina et al. (2006) con un intenso trabajo de campo (672 horas-persona) para evaluar la diversidad herpetofaunística en el ecotono potrero-borde-interior en fragmentos de selva en la zona de estudio, sólo detectaron 4 individuos de $C$. vulcani ( 3 en el interior y 1 en el borde). Los espacios muestreados (incluyendo el interior de fragmentos) no incluían un sistema de arroyos o ríos, lo cual pudo haber influido en la baja abundancia detectada, pese al importante esfuerzo de recolecta aplicado incluso en lugares conservados. Por otro lado, la mayor abundancia registrada durante la época seca, resalta la importancia de los sistemas riparios como refugio para los individuos de la especie durante periodos cálidos y secos. Asimismo, estos sistemas pueden actuar como reservorios de diversidad de una cantidad importante de fauna, como aves, mamíferos y escarabajos coprófagos, en un paisaje con severa transformación como el estudiado (Estrada, 2008). En este sentido, la asociación entre la presencia de $C$. vulcani y los cuerpos de agua es un elemento relevante a considerar en cualquier estrategia para conservar la especie en la región.

La distribución de tamaño (LHC) y de masa corporal 
de los ejemplares recolectados en este estudio puede ser una aproximación gruesa de la estructura de edades de la población. De ser así, se observa un reclutamiento importante al interior de los fragmentos y remanentes riparios, lo cual confirmaría la relevancia de tales elementos del paisaje tanto para la persistencia, como para la dispersión y reproducción de la especie en paisajes dominados por pastizales.

La distribución conocida de C. vulcani en la sierra está sesgada hacia el norte y parece estar influida por la cercanía con centros de población humana, caminos y particularmente por la ubicación de la Estación de Biología Los Tuxtlas. Este es un sesgo común en muchas especies de anfibios cuando se quiere analizar su distribución espacial (Pineda y Lobo, 2009). En el caso de la especie en estudio, al analizar la distribución altitudinal y horizontal a lo largo del volcán San Martín Tuxtla, resulta fácil prever que, dada la semejanza ambiental con el resto de la sierra, la distribución real de la especie podría ser prácticamente toda la sierra. La especie tendría entonces una importante capacidad para habitar en distintos tipos de vegetación. Sin embargo, dados los distintos niveles de deforestación y fragmentación a lo largo de la región (Guevara et al., 2004a; Arroyo-Rodríguez et al., 2009) es necesario confirmar su presencia en aquellos espacios no explorados, así como evaluar el estado que guardan sus poblaciones en otros ambientes como el bosque mesófilo o incluso en ambientes menos transformados como los cultivos de café con sombra, mango, plátano, aguacate o cítricos.

Finalmente, la sensibilidad de $C$. vulcani a la alteración del hábitat sugiere que la disminución en tamaño de los fragmentos de vegetación y el aumento en su aislamiento resultará en una disminución notable del número de individuos y poblaciones en la región. Si la transformación de bosques en potreros continúa en Los Tuxtlas, la persistencia a largo plazo de C. vulcani en la región esta comprometida.

\section{Agradecimientos}

A Julián Bueno, Elba M. López, Hernán Díaz, Maya Rocha, Ricardo Luria, Luis Vázquez, Itzue Caviedes, Daniel Aportela, Braulio y Juan Gómez, por su valiosa ayuda en campo. Luis Canseco-Márquez nos apoyó amablemente con la identificación de ejemplares recolectados, Karina Pineda ayudó con el manejo de imágenes y un revisor anónimo ofreció valiosas sugerencias al trabajo. A Rosamond Coates, por las facilidades otorgadas para el trabajo en campo. Al personal de la Estación de Biología Los Tuxtlas, Instituto de Biología, UNAM; a Sierra Nuyuxia A.C. y al Instituto de Ecología, A.C.; a IDEA
WILD, por la donación de equipo de campo. Este trabajo fue financiado por el Fondo Sectorial SEMARNATCONACYT (proyecto 23588) y el Fondo Mixto Gobierno del Estado de Veracruz-CONACYT (proyecto 34103).

\section{Literatura citada}

Arroyo-Rodríguez, V., E. Pineda, F. Escobar y J. BenítezMalvido. 2009. Value of small patches in the conservation of plant-species diversity in highly fragmented rainforest. Conservation Biology 23:729-739.

Castillo-Campos, G. y J. Laborde. 2004. La vegetación. In Los Tuxtlas, el paisaje de la sierra, S. Guevara S., J. Laborde y G. Sánchez-Ríos (eds.). Instituto de Ecología/Unión Europea, Xalapa, Veracruz. p. 231-269.

Campbell, J. A. y J. M. Savage. 2000. Taxonomic reconsideration of Middle American frogs of the Eleutherodactylus group (Anura: Leptodactylidae): a reconnaissance of subtle nuances among frogs. Herpetological Monographs 14:186-292.

Dirzo, R. y M. C. García. 1992. Rates of deforestation in Los Tuxtlas, a Neotropical area in Veracruz, Mexico. Conservation Biology 6:84-90.

Estrada, A. 2008. Fragmentación de la selva y agroecosistemas como reservorio de conservación de la fauna silvestre en Los Tuxtlas, México. In Evaluación y conservación de biodiversidad en paisajes fragmentados de Mesoamérica, C.A. Harvey y J.C. Sáenz (eds.). Instituto Nacional de Biodiversidad, Santo Domingo de Heredia, Costa Rica. p. 327-348.

Estrada, A. y R. Coates-Estrada. 1996. Tropical rain forest fragmentation and wild populations of primates at Los Tuxtlas, Mexico. International Journal of Primatology 17:759-783.

Frost, D. R. 2009. Amphibian species of the world: an online reference. Versión 5.3 (12 de febrero, 2009). American Museum of Natural History, New York. http://research. amnh.org/herpetology/amphibia/

Gardner, T. A., J. Barlow y C. A. Peres. 2007. Paradox, presumption and pitfalls in conservation biology: The importance of habitat change for amphibians and reptiles. Biological Conservation 138:166-179.

Gascon, C., T. E. Lovejoy, R. O. Bierregaard Jr., J. R. Malcolm, P. C. Stouffer, H. L. Vasconcelos, W. F. Laurance, B. Zimmerman, M. Tocher y S. Borges. 1999. Matrix habitat and species richness in tropical forest remnants. Biological Conservation 91:223-229.

Guevara, S. S., G. Sánchez-Ríos y R. Langrave. 2004a. La deforestación. In Los Tuxtlas, el paisaje de la sierra, S. Guevara S., J. Laborde D. y G. Sánchez-Ríos (eds.). Instituto de Ecología/Unión Europea, Xalapa, Veracruz. p. 85-110.

Guevara, S. S., J. Laborde y G. Sánchez-Ríos. 2004b. Los Tuxtlas, el paisaje de la sierra. Instituto de Ecología/Unión Europea, Xalapa, Veracruz. 287 p.

Hernández, O. O. 2005. Comparación de la comunidad de anfibios y reptiles entre zonas altas y bajas dentro de la reserva de la Estación de Biología, UNAM, Los Tuxtlas, Veracruz. Tesis, 
Facultad de Ciencias, Universidad Nacional Autónoma de México, México, D.F. 91 p.

Hijmans, R. J., L. Guarino, M. Cruz y E. Rojas. 2001. Computer tools for spatial analysis of plant genetic resources data: DIVA-GIS. Plant Genetic Resources Newsletter 127:15-19.

IUCN. 2008. 2008 IUCN Red list of threatened species. <www. iucnredlist.org $>$. Consultada el 23 de enero de 2009.

Lips, K. R., P. A. Burrowes, J. R. Mendelson III y G. Parra-Olea. 2005. Amphibian population declines in Latin America: a synthesis. Biotropica 37:222-226.

Lynch, J. D. 1965. A review of the rugulosus group of Eleutherodactylus in northern Central America. Herpetologica 21:102-113.

Pineda, E. y G. Halffter. 2004. Species diversity and habitat fragmentation: frogs in a tropical montane landscape in Mexico. Biological Conservation 117:499-508.

Pineda, E. y J. M. Lobo. 2009. Assessing the accuracy of species distribution models to predict amphibian species richness patterns. Journal of Animal Ecology 78:182-190.

Ramírez-Bautista, A. 1977. Algunos anfibios y reptiles de la región de "Los Tuxtlas" Veracruz. Tesis, Facultad de Biología, Universidad Veracruzana. Xalapa, Veracruz. $170 \mathrm{p}$.

Rasband, W. 1997. ImageJ v1.37. National Institutes of Health, USA. http://rsb.info.nih.govIij/

Salvatore, O. O. M. 2006. Diagnóstico de la estructura de las comunidades de anfibios y reptiles en la selva fragmentada de Los Tuxtlas. Tesis, Instituto de Biología, Universidad Nacional Autónoma de México, México, D.F. 83 p.

Santos-Barrera, G. 2004. Craugastor vulcani. In 2008 IUCN. Red list of threatened species. <www.iucnredlist.org>. Consultada el 23 de enero de 2009.

SEMARNAT (Secretaría del Medio Ambiente y Recursos Naturales). 2002. Norma Oficial Mexicana NOM-059ECOL-2001, Protección ambiental-Especies nativas de
México de flora y fauna silvestres-Categorías de riesgo y especificaciones para su inclusión, exclusión o cambio-Lista de especies en riesgo. Diario Oficial de la Federación, 6 de marzo, segunda sección.

Soto, E. M. 2004. El clima. In Los Tuxtlas, el paisaje de la sierra, S. Guevara S., J. Laborde D. y G. Sánchez-Ríos (eds.). Instituto de Ecología/Unión Europea, Xalapa, Veracruz. p. 195-199.

StatSoft, Inc. 2004. STATISTICA for Windows. Versión 7.0. Tulsa. http://www.statsoft.com.

Stuart, S. N., J. S. Chanson, N. A. Cox, B. E. Young, A. S. L. Rodrigues, D. L. Fischman y R. W. Waller. 2004. Status and Trends of Amphibian Declines and Extinctions Worldwide. Science 306:1783-1786.

Suazo-Ortuño, I., J. Alvarado-Díaz y M. Martínez-Ramos. 2008. Effects of conversion of dry tropical forest to agricultural mosaic on herpetofaunal assemblages. Conservation Biology 22:362-374.

Urbina-Cardona, J. N., M. Olivares-Pérez y V. H. Reynoso. 2006. Herpetofauna diversity and microenvironment correlates across a pasture-edge-interior ecotone in tropical rainforest fragments in the Los Tuxtlas Biosphere Reserve of Veracruz, Mexico. Biological Conservation 132:61-75.

Villar, R. M. 2007. Análisis de la composición y diversidad de anfibios y reptiles, en áreas con características de corredores biológicos en Los Tuxtlas, Veracruz. Tesis, Facultad de Ciencias, Universidad Nacional Autónoma de México. México, D.F. 98 p.

Vogt, R. C., B. J. L. Villareal y G. Pérez-Higareda. 1997. Lista anotada de anfibios y reptiles. In Historia Natural de Los Tuxtlas, E. González-Soriano, R. Dirzo y R. C. Vogt (eds.). Universidad Nacional Autónoma de México, México, D.F. p. 507-528.

Zar, J. 1999. Biostatistical analysis, 4a edición, Prentice Hall, Upper Saddle River, New Jersey. 929 p. 